\title{
Modification and de novo design of non-ribosomal peptide synthetases (NRPS) using specific assembly points within condensation domains
}

Kenan A. J. Bozhüyük ${ }^{1}$, Annabell Linck ${ }^{1}$, Andreas Tietze ${ }^{1}$, Frank Wesche ${ }^{1}$, Sarah Nowak $^{1}$, Florian Fleischhacker ${ }^{1}$, Helge B. Bode ${ }^{1,2}$ *

\section{${ }^{1}$ Fachbereich Biowissenschaften, Merck Stiftungsprofessur für Molekulare} Biotechnologie, Goethe-Universität Frankfurt, Frankfurt am Main 60438, Germany. ${ }^{2}$ Buchmann Institute for Molecular Life Sciences, Goethe-Universität Frankfurt, Frankfurt am Main 60438, Germany.

K.A.J.B. and A.L. contributed equally to this work.

*e-mail: h.bode@bio.uni-frankfurt.de

\section{Abstract}

Many important natural products are produced by non-ribosomal peptide synthetases (NRPSs) ${ }^{1}$.These giant enzyme machines activate amino acids in an assembly line fashion in which a set of catalytically active domains is responsible for the section, activation, covalent binding and connection of a specific amino acid to the growing peptide chain ${ }^{1,2}$. Since NRPS are not restricted to the incorporation of the 20 proteinogenic amino acids, their efficient manipulation would give access to a diverse range of peptides available biotechnologically. Here we describe a new fusion point inside condensation $(\mathrm{C})$ domains of NRPSs that enables the efficient production of peptides, even containing non-natural amino acids, in yields higher than $280 \mathrm{mg} / \mathrm{L}$. The technology called eXchange Unit $2.0\left(\mathrm{XU}_{2.0}\right)$ also allows the generation of targeted peptide libraries and therefore might be suitable for the future identification of bioactive peptide derivatives for pharmaceutical and other applications. 


\section{Introduction}

Secondary metabolite derived drugs have become essential agents to cure infectious diseases during the last almost 70 years $^{3,4}$. Yet, infectious diseases are still the second major cause of death worldwide and furthermore, the world is facing a global public-health crisis as there is a growing risk of re-entering a pre-antibiotic era, since more and more infections are caused by multi-drug-resistant bacteria ${ }^{5}$.

One source of new antibacterial agents are non-ribosomally made peptides (NRPs). Their high structural diversity imparts to them many properties of biological relevance and peptides have been identified with antibiotic, antiviral, anti-cancer, antiinflammatory, immunosuppressant and surfactant qualities ${ }^{6,7,8}$. However, natural products often need to be modified to improve clinical properties and/or bypass resistance mechanisms ${ }^{9,10}$. To date, most clinically used NP derivatives are created by means of semi-synthesis ${ }^{9,11}$. A promising alternative strategy is the use of engineering approaches to modify NRP producing non-ribosomal peptide synthetase (NRPS) directly in order to produce optimized or non-natural natural products ${ }^{12}$. However, to date most attempts to achieve this have yielded impaired or nonfunctional biosynthetic machineries ${ }^{7,13}$.

NRPSs are large multienzyme complexes (megasynthases) ${ }^{14}$ that form peptides not limited to the twenty proteinogenic amino acids $(A A)^{15}$. Furthermore, these NRPS can generate linear or cyclic peptides containing D-AA, N-methylated AA, N-terminal attached fatty acids (FA) or heterocycles ${ }^{1,2,14,15}$. NRPS do this by exhibiting a strict modular architecture in which a module is defined as the catalytic unit responsible for the incorporation of one specific building block (e.g. AA) into the growing peptide chain $(\mathrm{N} \rightarrow \mathrm{C})$ and associated functional group modifications ${ }^{16}$. Modules are composed of domains that catalyze the single reaction steps like activation, covalent binding, optional modification of the building blocks, and condensation with the amino 
acyl or peptidyl group on the neighboring module ${ }^{17}$. At least three domains or essential enzymatic activities, respectively, are necessary for the non-ribosomal production of peptides (Fig. 1). They reside in the adenylation (A) for AA activation, thiolation ( $\mathrm{T}$ ) for AA tethering, and condensation $(\mathrm{C})$ domains for peptide bond formation. Finally, most NRPS termination modules harbor a TE domain that releases the peptide, often in a cyclized form. These standard domains are additionally joined by tailoring domains that can catalyze epimerization $(E)$, methylation (MT), cyclization (CY) or other modifications of the building blocks or the growing peptide chain ${ }^{1}$.

Due to the modular character of the NRPS scientists strived to reprogram these systems via (I) the substitution of the A or paired A-T domain activating an alternative substrate, (II) the targeted alteration of just the substrate binding pocket of the $A$ domain or (III) substitutions that treat C-A or C-A-T domain units as inseparable pairs 7. These strategies are complemented by recombination studies which have sought to re-engineer NRPS by $\mathrm{T}^{18}, \mathrm{~T}-\mathrm{C}-\mathrm{A}^{19}$, communication domain ${ }^{20}$ and A-T-C swapping ${ }^{21}$. However, with exception of the latter and recently published strategy, denoted as the concept of exchange $\underline{\text { Units }}(\mathrm{XU})^{22}$, it has been difficult to develop clearly defined, reproducible and validated guidelines for engineering modified NRPS.

The limitation of the $\mathrm{XU}$-concept is that the natural downstream $\mathrm{C}$ domain specificity must be obeyed clearly restricting its applicability and the C-domain specificities have to be met - at the donor as well as at the acceptor site. This disadvantage can be accepted if a large number of XUs with different downstream $C$ domains are available. Due to these limitations also at least two XUs have to be exchanged to produce a new peptide derivative that differs in one AA position from the primary sequence of the wild type (WT) peptide ${ }^{22}$. However, a more flexible system reducing the limitations of C-domain specificities would drastically reduce the amount of NRPS 
building blocks necessary to produce or alter particular peptides and would enable the creation of artificial natural product libraries with hundreds or thousands of entities for large scale bioactivity screenings.

\section{Results and Discussion}

\section{$\underline{\text { C-domains have acceptor site substrate specificity }}$}

To verify the influence of the $\mathrm{C}$-domains acceptor site $\left(\mathrm{C}_{\mathrm{Asub}}\right)$ proof reading activity, the GameXPeptide producing NRPS GxpS of Photorhabdus luminescens TT01 (Supplementary Figure 1 and 2) was chosen as a model system ${ }^{23,24}$. A recombinant GxpS was constructed, not complying with the C-domain specificity rules of the XU concept $^{22}$. Here, XU2 of GxpS (Fig. 1b, NRPS-1) was exchanged against XU2 of the bicornutin producing NRPS (BicA, Fig. 1c) ${ }^{25}$. Although both XUs are Leu specific, they are differentiated by their $\mathrm{C}_{\text {Asub }}$ specificities - Phe for XU2 of GxpS and Arg for XU2 of BicA. Therefore, no peptide production was observed as expected. This experiment confirmed previously published scientific results from in vitro experiments ${ }^{26-29}$, and illustrates that $C$ domains indeed are highly substrate specific at their $C_{\text {Asub. }}$. From the available structural data of $\mathrm{C}$ domains it is clear that they show a pseudodimer configuration ${ }^{28,30-32}$ with their catalytic center, including the HHXXXDG motif, having two binding sites - one for the electrophilic donor substrate and one for the nucleophilic acceptor substrate ${ }^{29}$ (Fig. 1a and Supplementary Figure 3). Therefore we concluded that the four AA long conformationally flexible loop/linker between both subdomains might be the ideal target to reconfigure $\mathrm{C}$ domain specificities via the engineering of $\mathrm{C}$ domain hybrids (Fig. 1a). For this purpose the Arg specific $\mathrm{C}_{\text {Asub }}$ of the GxpS-BicA hybrid NRPS (Fig. 1b, NRPS-1) was re-exchanged to the Leu specific $\mathrm{C}_{\text {Asub }}$ of GxpS, restoring the functionality of the hybrid NRPS (NRPS-2) and leading to the production of GameXPeptide A-D (1-5) in $217 \%$ (107 mg/L) yield compared to 
the WT GxpS (Fig. 1b) as confirmed by MS/MS analysis and comparison of the retention times with a synthetic standard (Supplementary Figure 4).

\section{The eXchange Unit 2.0 concept}

From these results in conjunction with bioinformatics analysis, we concluded that Cdomains acceptor and donor site $\left(\mathrm{C}_{\text {Dsub }}\right)$ mark a self-contained catalytically active unit $\mathrm{C}_{\text {Asub }}-\mathrm{A}-\mathrm{T}-\mathrm{C}_{\text {Dsub }} \quad\left(\mathrm{XU}_{2.0}\right) \quad$ without interfering major domain-domain interfaces/interactions during the NRPS catalytic cycle ${ }^{33}$. In order to validate the proposed $\mathrm{XU}_{2.0}$ building block (Fig. 1c) and to compare the production titers with a natural NRPS, we reconstructed GxpS (Fig. 1b) in two variants (Fig. 2a, NRPS-3 and -4). Each from five $X_{2.0}$ building blocks from four different NRPSs (XtpS, AmbS, GxpS, GarS, HCTA) (Supplementary Figure 5):

NRPS-3 showed a mixed $C / E_{\text {Dsub }}-C_{\text {Asub }}$-domain between $\mathrm{XU}_{2.0} 3$ and $\mathrm{XU}_{2.0} 4$ (Fig. 2a), to reveal if $\mathrm{C}$ and $\mathrm{C} / \mathrm{E}$ domains can be combined. In NRPS-4 $\mathrm{XU}_{2.0} 3$ from HCTA instead of GarS was used in order to prevent any incompatibilities (Fig. 2a).

Whereas NRPS-3 (Fig. 2a) showed no detectable production of any peptide, NRPS-4 (Fig. 2) resulted in the production of 1 and 3 in 66 and $6 \%$ yield, respectively, compared to the natural GxpS, as confirmed by MS/MS analysis and comparison of the retention times of synthetic standards (Fig. 2a, Supplementary Figure 6). In line with expectations from domain sequences, phylogenetics, as well as structural idiosyncrasies of C/E- and C-domains ${ }^{29}$, it may be deduced from these results that C/E and C-domains cannot be combined with each other. Although NRPS-4 (Fig. 2a) showed moderately reduced production titers, most likely due to the non-natural $\mathrm{C}_{\text {Dsub }}-\mathrm{C}_{\text {Asub }}$ pseudo-dimer interface, the formal exchange of the promiscous $\mathrm{XU}_{2.0} 1$ from GxpS (for Val/Leu) against the Val-specific $X_{2.0} 1$ from XtpS led to exclusive production of 1 and 3 (Fig. 2a) without production of 2 and 4 observed in the original 
GxpS (Fig. 1b), indicating that the $\mathrm{XU}_{2.0}$ can also be used to increase product specificity and to reduce the formation of side products.

Additional GameXPeptide derivatives were generated (Fig. 2a, NRPS-5) by combining building blocks according to the definition of $\mathrm{XU}^{22}$ and $\mathrm{XU}_{2.0}$. Three

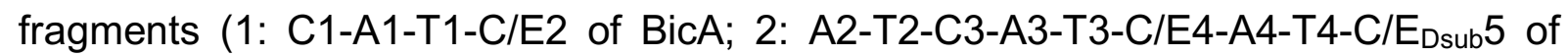
GxpS; 3: C/E Asub5-A5-T5-C term $_{\text {of }}$ BicA) from two NRPSs (BicA: Xenorhabdus budapestensis DSM 16342; GxpS: Photorhabdus luminescens TT01) were used as building blocks ${ }^{23,25}$. The expected two Arg containing cyclic pentapeptides 6 and 7 were produced in yields of 4.6 and $0.4 \mathrm{mg} / \mathrm{L}$ and were structurally confirmed by chemical synthesis (Supplementary Figure 7). Both peptides only differ in Leu or Phe at position three from the promiscous $\mathrm{XU}_{2.0} 3$ from $\mathrm{GxpS}$. Despite a drop of production rate in comparison to the WT NRPS, we successfully demonstrated that the recently published $\mathrm{XU}^{22}$ as well as the novel $\mathrm{XU}_{2.0}$ strategy can be combined for successful reprogramming of NRPS and the production of tailor-made peptides.

To show the general applicability of the novel $\mathrm{XU}_{2.0}$ building block an artificial NRPS was designed de novo from building blocks of Gram-positive origin (NRPS for the production of bacitracin ${ }^{34}$ from Bacillus licheniformis ATCC 10716 and surfactin ${ }^{35}$ from Bacillus subtilis MR 168), since all aforementioned recombined NRPS are of Gram-negative origin. The expected pentapeptide 8 containing the bacitracin NRPS derived thiazoline ring was produced in yields of $21.09 \mathrm{mg} / \mathrm{L}$ (Fig. 2b, Supplementary Figure 8) in E. coli, showing the universal nature of the $\mathrm{XU}_{2.0}$.

\section{Amending the starter unit}

Up to date there is no publication describing the successful exchange of a starter unit against an internal NRPS-fragment. Reasons for that might be that (I) starter-Adomains in general comprise some kind of upstream sequence of variable length with 
unknown function and structure, which makes it difficult to define an appropriate artificial leader sequence, and that (II) necessary interactions at the C-A interface may be important for adenylation activity and A-domain stability as indicated recently 36,37. In order to test whether the $\mathrm{XU}_{2.0}$ concept can also be applied to modify starter units, three recombinant GxpS constructs (NRPS-7 - 9) with internal domains as starting units were created (Fig. 2c). In NRPS-7 A1-T1-C Dsub2 of GxpS was exchanged against $\mathrm{C} 2 \mathrm{~A} 3-$ linker-A3-T3- $\mathrm{C}_{\text {Dsub }} 4$ of $\mathrm{XtpS}$ since all starter $\mathrm{A}$-domains have at least a preceding C-A linker sequence. Since there are several examples of NRPSs carrying catalytically inactive starter C-domains (e.g. AmbS) ${ }^{38}, \mathrm{~A} 1-\mathrm{T} 1-\mathrm{C}_{\text {Dsub }}$ of GxpS was altered to C3-A3-T3-C Dsub4 of XtpS in NRPS-8. In NRPS-9 A1-T1$\mathrm{C}_{\text {Dsub2 }}$ of $\mathrm{GxpS}$ was altered to $\mathrm{C}_{\text {Asub }}-\mathrm{A} 3-\mathrm{T} 3-\mathrm{C}_{\text {Dsub}} 4$ of $\mathrm{Xtps}$ since there are natural NRPSs exhibiting parts of a C-domain (e.g. BicA) in front of the starter A-domain.

Whereas NRPS-7 (Fig. 2c) did not show production of the desired peptides, NRPS-8 and NRPS-9 synthesized 1 and 3 in yields between $0.31-0.44 \mathrm{mg} / \mathrm{L}$ (Fig. 2c, Supplementary Figure 9). This indicates that internal A-domains can indeed be used as starter domains, if the upstream $\mathrm{C}_{\text {Asub }}$ or $\mathrm{C}$-domain is kept in front of the A-domain pointing to the importance of a functional C-A interface for A-domain activity. Yet, the observed low production titers might indicate that for example the observed difference in codon usage and the lower GC-content at the beginning of WT NRPS encoding genes could have a major impact on transcriptional and/or translational efficiency in conjunction with protein folding as described previously ${ }^{39,40}$.

\section{Production of functionalized peptides}

Besides simply creating NRP derivatives, one useful application of NRPS reprogramming is the incorporation of non-proteinogenic or even non-natural $A A$. Examples for the latter might be AAs containing alkyne or azide groups, allowing 
reactions like $\mathrm{Cu}(\mathrm{I})$-catalyzed or strain-promoted Huisgen cyclization also known as "click" reactions $41,42,42-44$. Yet, although NPRS and A domains have been examined exhaustively for several years, no general method for the in vivo functionalization of NRPs are available by reprogramming NRPS templates.

A broad range of AAs are accepted by the A3 domain of GxpS (Supplementary Figure 10) resulting in a large diversity of natural GameXPeptides ${ }^{23,24}$. Moreover, by using a $\gamma^{-18} \mathrm{O}_{4}$-ATP pyrophosphate exchange assay for A domain activity ${ }^{45,46}$ and adding substituted phenylalanine derivatives to $E$. coli cultures expressing GxpS, the respective A3-domain was identified as being able to activate (in vitro, Supplementary Figure 10) and incorporate (in vivo, Supplementary Figure 11) several ortho- $(o)$, meta- $(m)$ and para- $(p)$ substituted phenylalanine derivatives, including 4-azido-L-phenylalanine $\left(p \mathrm{~N}_{3}-\mathrm{F}\right)$ and O-propargyl-L-tyrosine $(¥-Y)$. When the Val specific $X_{2.03}$ of the xenotetrapeptide ${ }^{47}$ (9) (Supplementary Figure 12) producing NRPS (XtpS) from $X$. nematophila HGB081 was exchanged against $\mathrm{XU}_{2.0} 3$ of GxpS, six new xenotetrapeptide derivatives (10-15) in yields between 0.17-106 $\mathrm{mg} / \mathrm{L}$ were produced reflecting its natural promiscuity (Fig. 3, Supplementary Figure 13). After adding $p N_{3}-F$ and $¥-Y$ to growing $E$. coli cultures expressing recombined XtpS (NRPS-10), six functionalized peptides (16-21) differing in position 3 were produced in yields of 5-228 mg/L with 17,18 and 19 being structurally confirmed by chemical synthesis. Moreover, although the A4-domain of XtpS shows an exclusive specificity for Val in the WT NRPS XtpS, peptides 11, 13, 16 and 21 produced by NRPS-10 additionally incorporate Leu at position four. The observed change in specificity might be due to the hybrid C/E4-domain upstream of A4 from NRPS-10. Leu is the original substrate downstream of the introduced $X U_{2.0} 3$ of GxpS (Fig. 1b) in its natural context, indicating that the overall structure of $C$ domains along with resulting transformed $\mathrm{C}-\mathrm{A}$ interface interactions might influence the $\mathrm{A}$ domain 
substrate specificity. Recently, similar but in vitro observed effects were reported regarding $A$ domains from sulfazecin ${ }^{36}$ and microcystin ${ }^{37}$. This effect could also be used to increase the specificity of $A$ domains to prevent the formation of side products. Further investigations will shed light on this remarkable and yet unreported effect.

\section{Production of peptide libraries}

Modern drug-discovery approaches often apply the screening of compound libraries including NP libraries ${ }^{48}$ since they exhibit a wide range of pharmacophores, structural diversity and have the property of metabolite-likeness often providing a high degree of bioavailability. Yet, the NP discovery process is as expensive as time consuming ${ }^{49}$. Consequently, for bioactivity screenings the random recombination of certain NRPS fragments would be a powerful tool to create focused artificial NP-like libraries.

In an initial test, GxpS was chosen for the generation of a focused peptide library created via a one-shot yeast based TAR cloning approach ${ }^{38,50}$. Here, the third position of the peptide (D-Phe) was randomized (Fig. 4a) using six unique $X_{2.0}$ building blocks from six NRPS (KolS ${ }^{51}, \mathrm{AmbS}_{\mathrm{mir}}{ }^{38}, \mathrm{Pax}^{52}, \mathrm{AmbS}_{\text {ind, }} \mathrm{XIIS}$; for detail see Supplementary Figure 5), resulting in the production of $\mathbf{1}$ and four new GameXPeptide derivatives (22-25) in yields of 3-92 $\mathrm{mg} / \mathrm{L}$ that were structurally confirmed by chemical synthesis (Supplementary Figure 14).

For the generation of a second and structurally more diverse peptide library, positions 1 (D-Val) and 3 (D-Phe) of GxpS were selected in parallel for randomization (Fig. 4b). From the experimental setup theoretically 48 different cyclic or linear peptides could be expected. Screening of $50 \mathrm{E}$. coli clones resulted in the identification of 18 unique cyclic and linear peptides $(1,5,11,13,24,26-36)$ from four peptide producing clones differing in peptide length and AA composition (Supplementary Figure 15). Since only 
7 from 18 identified peptides belong to the originally expected set of peptides, homologues recombination in yeast based reprogramming of NRPS also allows the production of unexpected peptides due to unexpected homologues recombination events resulting in an additional layer of peptide diversification, as observed previously ${ }^{22}$.

Randomizing directly adjacent positions via a similar approach requires a standardized nucleotide sequence (40 base pairs) for homologues recombination (Supplementary Figure 16) ${ }^{38,50}$. From a detailed analysis of the T-C didomain crystal structure of TycC5-6 (PDB-ID: 2JGP), helix a5 (I253-F265) next to the C domain's pseudo-dimer linker was identified as an ideal target for homologues recombination. Subsequently, an artificial a5 helix was designed to randomize position 2 (L-Leu) and 3 (D-Phe) of GxpS (Supplementary Figure 16a), being an integral part of all resulting recombinant $\mathrm{C} 3$ domains and therefore connecting $\mathrm{XU}_{2.0} 2$ and 3 . The applied $\alpha 5$ helix was defined as the consensus sequence of all involved $\mathrm{XU}_{2.0}$ building blocks (Supplementary Figure 16b). Screening of 25 E. coli clones revealed the synthesis of eight cyclic and linear GameXPeptides (1, 23-24, 31, 34, 37-39) from three peptide producing clones in good yields, showing the general applicability of redesigning a5 with respect to randomly reprogramming biosynthetic templates (Fig. 4c and Supplementary Figure 17).

\section{$\underline{\text { Conclusion }}$}

We have recently published the $\mathrm{XU}$ concept enabling the efficient reprogramming of NRPS but limited in its applicability by downstream C domain specificities. Here we present the $\mathrm{XU}_{2.0}$ concept that eliminates these limitations by a direct assembly inside the $\mathrm{C}$ domains and allows the production of natural and artificial peptides in yields up to $280 \mathrm{mg} / \mathrm{L}$. For the construction of any peptide based on the 20 
proteinogenic AAs only $80 \mathrm{XU}_{2.0}$ building blocks are necessary (only four of each: $\mathrm{C}_{\text {Dsub }}-\mathrm{A}-\mathrm{T}-\mathrm{C}_{\text {Asub }}, \quad \mathrm{C}_{\text {Dsub }}-\mathrm{A}-\mathrm{T}-\mathrm{C} / \mathrm{E}_{\text {Asub }}, \quad \mathrm{C} / \mathrm{E}_{\text {Dsub }}-\mathrm{A}-\mathrm{T}-\mathrm{C} / \mathrm{E}_{\text {Asub }}, \quad$ and $\left.\quad \mathrm{C} / \mathrm{E}_{\text {Dsub }}-\mathrm{A}-\mathrm{T}-\mathrm{C}_{\text {Asub }}\right)$ whereas 800 building blocks would be necessary to generate the same number of peptides using the $\mathrm{XU}$ concept. Consequently, the introduction of the $\mathrm{XU}_{2.0}$ simplifies and broadens the possibilities of biotechnological applications with respect to optimize bioactive agents via NRPS engineering exemplified for the reprogramming of NRPSs (Fig. 1 and 2) or the production of functionalized peptides by incorporating $\mathrm{XU}_{2.0}$ building blocks accepting non-natural AAs like $p \mathrm{~N}_{3}-\mathrm{F}$ and $¥-\mathrm{Y}$ (Fig. 3, Supplementary Figure 9) allowing further derivatization $41,42,53$.

However, the true strength of the $\mathrm{XU}_{2.0}$ concept is its application to generate random NP-like peptide libraries (Fig. 4) for subsequent bioactivity screenings. The possible automation of NRPS library design coupled to a bioactivity screening opens up entirely new opportunities of identifying novel lead compounds in the future. Especially in the area of anti-infective research the $\mathrm{XU}_{2.0}$ concept might allow a fast access to natural product derivatives with altered bioactivity profiles or for the generation of producer strains with less side products to facilitate compound purification.

Supplementary Information is available in the online version of the paper.

Acknowledgements The authors are grateful to Melanie Lindner and Caspar Zizka for help with the construction of selected constructs and Dr. Carsten Kegler for helpful discussions. This work was funded in part by a European research starting grant under grant agreement no. 311477 and LOEWE program of the state of Hesse as part of the MegaSyn research cluster. H.B.B. acknowledges the Deutsche Forschungsgemeinschaft for funding of the Impact II qTof mass spectrometer (INST 
161/810-1)

Author Contributions K.A.J.B. and H.B.B. designed the experiments. K.A.J.B., A.L., A.T. and S.N. performed all molecular biology and biochemical experiments, F.W. synthesized all peptide standards that were used for HPLC/MS-based quantification performed by A.L. All authors analysed the results and K.A.J.B., A.L. and H.B.B. wrote the manuscript. All authors saw and approved the manuscript.

\section{References}

1. Süssmuth, R. D. \& Mainz, A. Nonribosomal Peptide Synthesis-Principles and Prospects. Angewandte Chemie International Ed. 56, 3770-3821 (2017).

2. Sieber, S. A. \& Marahiel, M. A. Molecular mechanisms underlying nonribosomal peptide synthesis. Approaches to new antibiotics. Chemical Reviews 105, 715738 (2005).

3. Clardy, J., Fischbach, M. A. \& Walsh, C. T. New antibiotics from bacterial natural products. Nature Biotechnology 24, 1541-1550 (2006).

4. Nussbaum, F. von, Brands, M., Hinzen, B., Weigand, S. \& Häbich, D. Antibacterial natural products in medicinal chemistry - exodus or revival? Angewandte Chemie International Ed. 45, 5072-5129 (2006).

5. Nathan, C. Antibiotics at the crossroads. Nature 431, 899-902 (2004).

6. Felnagle, E. A. et al. Nonribosomal peptide synthetases involved in the production of medically relevant natural products. Molecular Pharmaceutics 5, 191-211 (2008).

7. Calcott, M. J. \& Ackerley, D. F. Genetic manipulation of non-ribosomal peptide synthetases to generate novel bioactive peptide products. Biotechnology Letters 36, 2407-2416 (2014).

8. Sieber, S. A. \& Marahiel, M. A. Molecular mechanisms underlying nonribosomal peptide synthesis. Approaches to new antibiotics. Chemical Reviews 105, 715738 (2005).

9. O'Connell, K. M. G. et al. Combating multidrug-resistant bacteria. Current strategies for the discovery of novel antibacterials. Angewandte Chemie International Ed. 52, 10706-10733 (2013).

10.Bush, K. Improving known classes of antibiotics. An optimistic approach for the future. Current Opinion in Pharmacology 12, 527-534 (2012).

11.Kirschning, A. \& Hahn, F. Merging chemical synthesis and biosynthesis. A new chapter in the total synthesis of natural products and natural product libraries. Angewandte Chemie International Ed. 51, 4012-4022 (2012). 
12.Baltz, R. H. Combinatorial biosynthesis of cyclic lipopeptide antibiotics. A model for synthetic biology to accelerate the evolution of secondary metabolite biosynthetic pathways. ACS Synthetic Biology 3, 748-758 (2014).

13.Winn, M., Fyans, J. K., Zhuo, Y. \& Micklefield, J. Recent advances in engineering nonribosomal peptide assembly lines. Natural Product Reports 33, 317-347 (2016).

14.Grünewald, J. \& Marahiel, M. A. Chemoenzymatic and template-directed synthesis of bioactive macrocyclic peptides. Microbiology and molecular biology reviews 70, 121-146 (2006).

15.Caboche, S., Leclère, V., Pupin, M., Kucherov, G. \& Jacques, P. Diversity of monomers in nonribosomal peptides. Towards the prediction of origin and biological activity. Journal of Bacteriology 192, 5143-5150 (2010).

16.Cane, D. E. Harnessing the Biosynthetic Code. Combinations, Permutations, and Mutations. Science 282, 63-68 (1998).

17.Mootz, H. D., Schwarzer, D. \& Marahiel, M. A. Ways of Assembling Complex Natural Products on Modular Nonribosomal Peptide Synthetases A list of abbreviations can be found at the end of the text. ChemBioChem 3, 490 (2002).

18.Beer, R. et al. Creating functional engineered variants of the single-module nonribosomal peptide synthetase IndC by T domain exchange. Molecular BioSystems 10, 1709-1718 (2014).

19. Calcott, M. J. \& Ackerley, D. F. Portability of the thiolation domain in recombinant pyoverdine non-ribosomal peptide synthetases. BMC Microbiology 15, 162 (2015).

20.Chiocchini, C., Linne, U. \& Stachelhaus, T. In vivo biocombinatorial synthesis of lipopeptides by COM domain-mediated reprogramming of the surfactin biosynthetic complex. Chemistry \& Biology 13, 899-908 (2006).

21.Duerfahrt, T., Doekel, S., Sonke, T., Quaedflieg, P. J. L. M. \& Marahiel, M. A. Construction of hybrid peptide synthetases for the production of alpha-I-aspartyl-Iphenylalanine, a precursor for the high-intensity sweetener aspartame. Eur J Biochem 270, 4555-4563 (2003).

22.Bozhüyük, K. A. J. et al. De novo design and engineering of non-ribosomal peptide synthetases. Nature Chemistry 5, 191 (2017). doi: 10.1038/NCHEM.2890.

23.Bode, H. B. et al. Determination of the absolute configuration of peptide natural products by using stable isotope labeling and mass spectrometry. Chemistry Eur $J$ 18, 2342-2348 (2012).

24.Nollmann, F. I. et al. Insect-specific production of new GameXPeptides in Photorhabdus luminescens TTO1, widespread natural products in entomopathogenic bacteria. ChemBioChem 16, 205-208 (2015).

25.Fuchs, S. W. et al. Neutral loss fragmentation pattern based screening for arginine-rich natural products in Xenorhabdus and Photorhabdus. Analytical Chemistry 84, 6948-6955 (2012).

26. Belshaw, P. J. Aminoacyl-CoAs as Probes of Condensation Domain Selectivity in Nonribosomal Peptide Synthesis. Science 284, 486-489 (1999). 
27.Susan L. Clugston et al. Chirality of Peptide Bond-Forming Condensation Domains in Nonribosomal Peptide Synthetases: The C5 Domain of Tyrocidine Synthetase Is a DCL Catalyst. Biochemistry 42, 12095-12104 (2003).

28.Samel, S. A., Schoenafinger, G., Knappe, T. A., Marahiel, M. A. \& Essen, L.-O. Structural and functional insights into a peptide bond-forming bidomain from a nonribosomal peptide synthetase. Structure 15, 781-792 (2007).

29.Rausch, C., Hoof, I., Weber, T., Wohlleben, W. \& Huson, D. H. Phylogenetic analysis of condensation domains in NRPS sheds light on their functional evolution. BMC Evolutionary Biology 7, 78 (2007).

30.Keating, T. A., Marshall, C. G., Walsh, C. T. \& Keating, A. E. The structure of $\mathrm{VibH}$ represents nonribosomal peptide synthetase condensation, cyclization and epimerization domains. Nature Structural Biology 9, 522-526 (2002).

31.Tanovic, A., Samel, S. A., Essen, L.-O. \& Marahiel, M. A. Crystal structure of the termination module of a nonribosomal peptide synthetase. Science 321, 659-663 (2008).

32.Bloudoff, K., Rodionov, D. \& Schmeing, T. M. Crystal structures of the first condensation domain of CDA synthetase suggest conformational changes during the synthetic cycle of nonribosomal peptide synthetases. Journal of Molecular Biology 425, 3137-3150 (2013).

33. Marahiel, M. A. A structural model for multimodular NRPS assembly lines. Natural Product Reports 33, 136-140 (2016).

34.Konz, D., Klens, A., Schörgendorfer, K. \& Marahiel, M. A. The bacitracin biosynthesis operon of Bacillus licheniformis ATCC 10716. Molecular characterization of three multi-modular peptide synthetases. Chemistry \& Biology 4, 927-937 (1997).

35. Cosmina, P. et al. Sequence and analysis of the genetic locus responsible for surfactin synthesis in Bacillus subtilis. Mol Microbiol 8, 821-831 (1993).

36.Li, R., Oliver, R. A. \& Townsend, C. A. Identification and Characterization of the Sulfazecin Monobactam Biosynthetic Gene Cluster. Cell Chemical Biology 24, 24-34 (2017).

37. Meyer, S. et al. Biochemical Dissection of the Natural Diversification of Microcystin Provides Lessons for Synthetic Biology of NRPS. Cell Chemical Biology 23, 462-471 (2016).

38.Schimming, O., Fleischhacker, F., Nollmann, F. I. \& Bode, H. B. Yeast homologous recombination cloning leading to the novel peptides ambactin and xenolindicin. ChemBioChem 15, 1290-1294 (2014).

39.Deb Roy, A., Grüschow, S., Cairns, N. \& Goss, R. J. M. Gene expression enabling synthetic diversification of natural products. Chemogenetic generation of pacidamycin analogs. Journal of the American Chemical Society 132, 1224312245 (2010).

40.Goodman, D. B., Church, G. M. \& Kosuri, S. Causes and effects of N-terminal codon bias in bacterial genes. Science 342, 475-479 (2013).

41.Sletten, E. M. \& Bertozzi, C. R. Bioorthogonal chemistry. Fishing for selectivity in a sea of functionality. Angewandte Chemie International Ed. 48, 6974-6998 (2009). 
42.Kolb, H. C. \& Sharpless, K.B. The growing impact of click chemistry on drug discovery. Drug Discovery Today 8, 1128-1137 (2003).

43.Pérez, A. J., Wesche, F., Adihou, H. \& Bode, H. B. Solid-Phase Enrichment and Analysis of Azide-Labeled Natural Products. Fishing Downstream of Biochemical Pathways. Chemistry Eur J 22, 639-645 (2016).

44.Pérez, A. J. \& Bode, H. B. "Click Chemistry" for the Simple Determination of FattyAcid Uptake and Degradation. Revising the Role of Fatty-Acid Transporters. ChemBioChem 16, 1588-1591 (2015).

45.Kronenwerth, M. et al. Characterisation of taxlllaids A-G; natural products from Xenorhabdus indica. Chemistry Eur J 20, 17478-17487 (2014).

46.Phelan, V. V., Du, Y., McLean, J. A. \& Bachmann, B. O. Adenylation enzyme characterization using gamma -(18)O(4)-ATP pyrophosphate exchange. Chemistry \& Biology 16, 473-478 (2009).

47.Kegler, C. et al. Rapid determination of the amino acid configuration of xenotetrapeptide. ChemBioChem 15, 826-828 (2014).

48. Harvey, A. L., Edrada-Ebel, R. \& Quinn, R. J. The re-emergence of natural products for drug discovery in the genomics era. Nature Reviews Drug Discovery 14, 111-129 (2015).

49.Lefevre, F. et al. Drugs from hidden bugs. Their discovery via untapped resources. Research in Microbiology 159, 153-161 (2008).

50.Gietz, R. D. \& Schiestl, R. H. Frozen competent yeast cells that can be transformed with high efficiency using the LiAc/SS carrier DNA/PEG method. Nature Protocols 2, 1-4 (2007).

51.Bode, H. B. et al. Structure Elucidation and Activity of Kolossin A, the D-/LPentadecapeptide Product of a Giant Nonribosomal Peptide Synthetase. Angewandte Chemie International Ed. 54, 10352-10355 (2015).

52.Fuchs, S. W., Proschak, A., Jaskolla, T. W., Karas, M. \& Bode, H. B. Structure elucidation and biosynthesis of lysine-rich cyclic peptides in Xenorhabdus nematophila. Organic \& Biomolecular Chemistry 9, 3130-3132 (2011).

53. Kries, H. et al. Reprogramming nonribosomal peptide synthetases for "clickable" amino acids. Angewandte Chemie International Ed. 53, 10105-10108 (2014). 


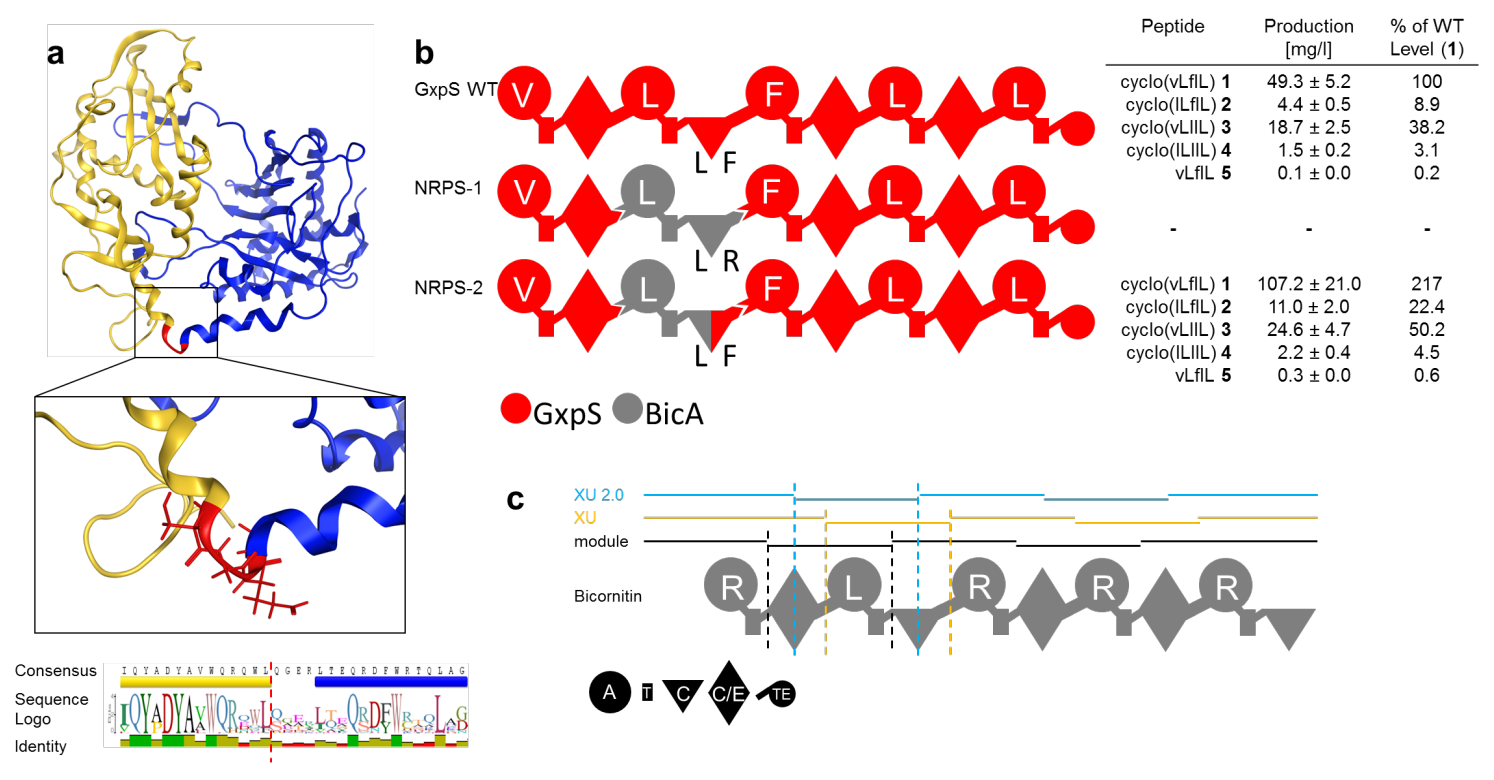

Figure 1. Modulation of $\mathbf{C}$-domain substrate specificity. (a) C-domain excised from the T-C bidomain TycC 5-6 from tyrocidine syntethase (TycC) of Brevibacillus brevis (PDB-ID: 2JGP) with N-terminal (yellow) and C-terminal (blue) subdomains depicted in ribbon representation (top). Boxed: enlarged representation of the $\mathrm{C}_{\mathrm{Dsub}}-$ $\mathrm{C}_{\text {Asub }}$ linker with contributing linker $\mathrm{AAs}$ in stick representation and fusion site marked in red. Bottom: sequence logo of $\mathrm{C}_{\text {Dsub }}-\mathrm{C}_{\text {Asub }}$ linker sequences from Photorhabdus and Xenorhabdus. (b) Schematic representation of WT GxpS, recombinant NRPS-1 and -2 as well as corresponding peptide yields as obtained from triplicate experiments. For peptide nomenclature the standard one letter AA code with lowercase for D-AA is used. (c) Schematic representation of BicA with modules and eXchange Units ( $X U$ and $\left.X U_{2.0}\right)$ highlighted. Specificities are assigned for all $A$ domains. For domain assignment the following symbols are used: A (large circles), T (rectangle), C (triangle), C/E (diamond), TE (C-terminal small circle). 


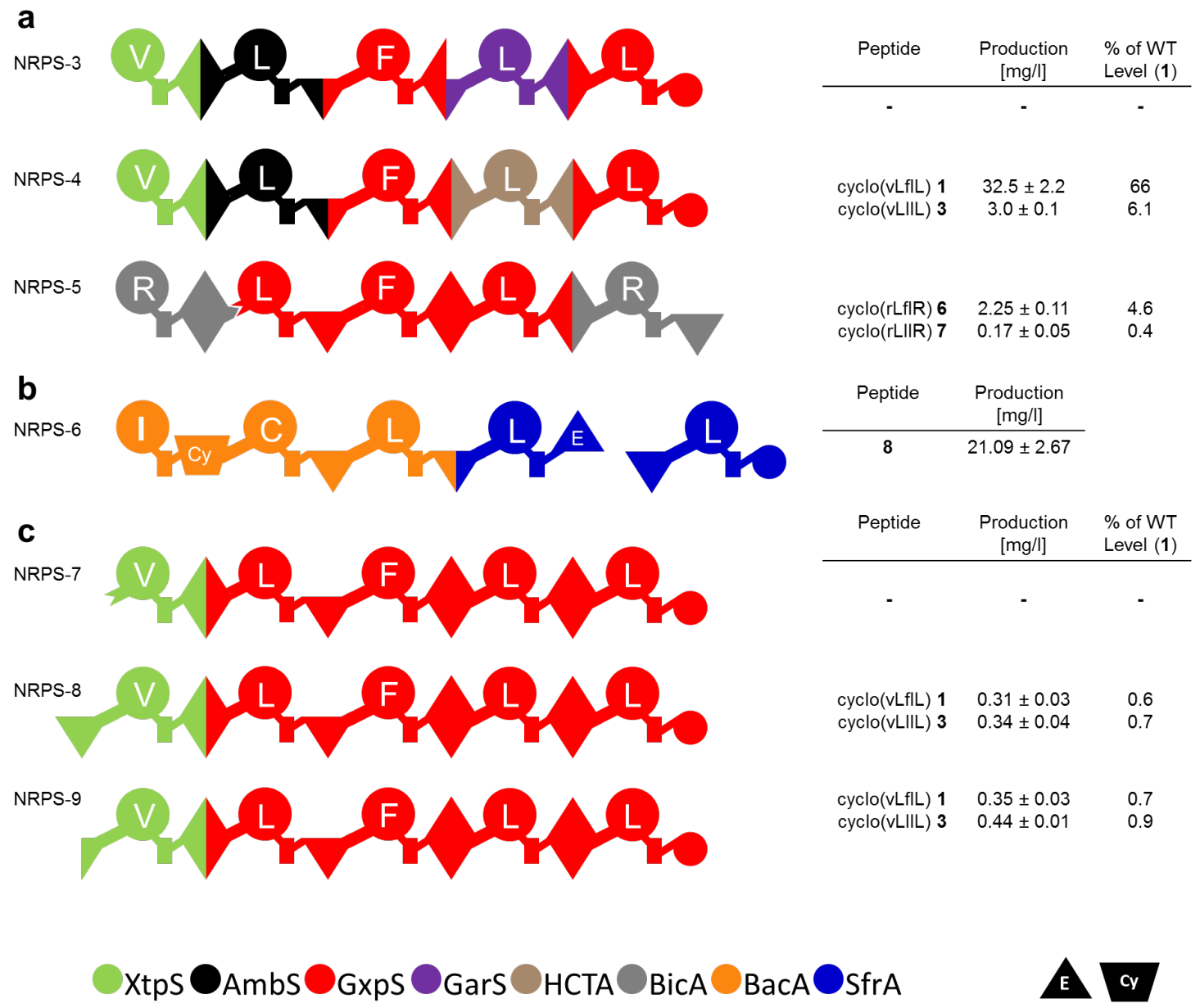

Figure 2. Design of recombinant NRPS for peptide production. (a) Generated recombinant GxpS (NRPS-3 - -5) and corresponding amounts of GameXPeptide derivatives 1, 3, 6, and 7 as determined in triplicates. (b) Recombinant NRPS-6 synthesizing 8. Building blocks are of Gram-positive origin. (c) Schematic representation of recombinant GxpS (NRPS-7 - -9) and corresponding peptide yields as obtained from triplicate experiments. For peptide nomenclature the standard $A A$ one letter code with lowercase for D-AA is used. For assignment of domain symbols see Fig. 1; further symbols are $\mathrm{E}$ (epimerization; inverted triangle), $\mathrm{CY}$ (heterocyclization; trapezium). Bottom: Color code of NRPS used as building blocks (for details see Supplementary Figure 5). 


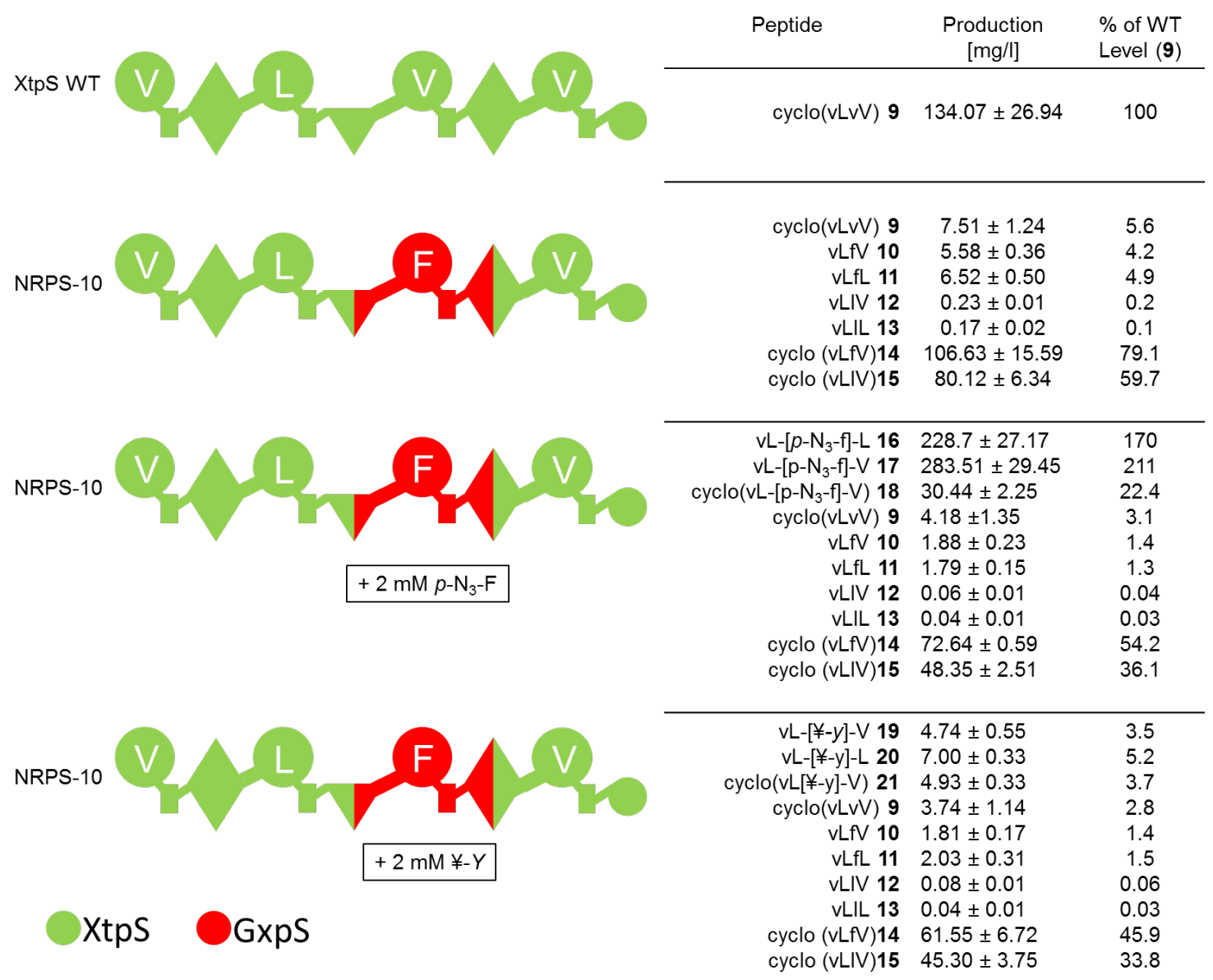

Figure 3. Creation of functionalized xenotetrapeptide derivatives. Schematic representation of WT XtpS, recombinant NRPS-10 and corresponding peptide yields as obtained from triplicate experiments. For peptide nomenclature the standard one letter AA code with lowercase for D-AA is used. For assignment of domain symbols see Fig. 1. Bottom: Color code of NRPS used as building blocks (for details see Supplementary Figure 5). 


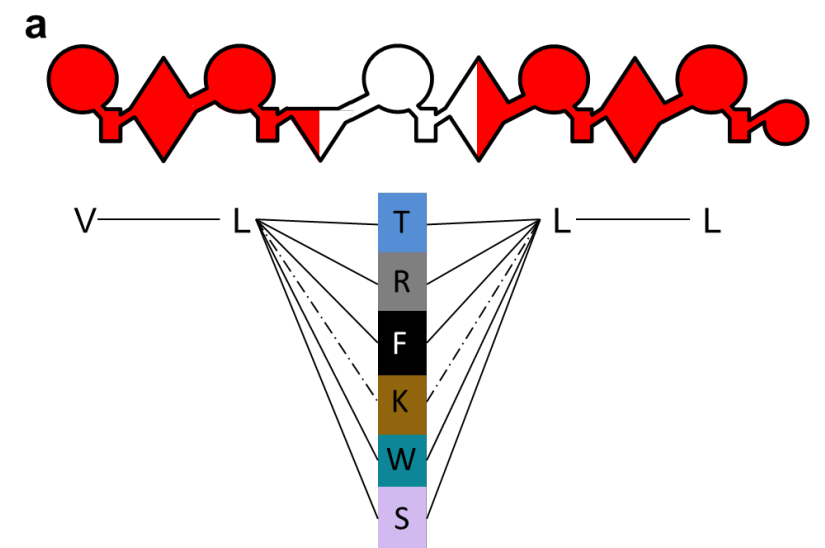

\begin{tabular}{lcc}
\multicolumn{1}{c}{ Peptide } & $\begin{array}{c}\text { Production } \\
{[\mathrm{mg} / \mathrm{l}]}\end{array}$ & $\begin{array}{c}\text { \% of WT } \\
\text { Level (1) }\end{array}$ \\
\hline cyclo(vLtL) 22 & $22.52 \pm 0.98$ & 46 \\
\hline cyclo(vLrlL) 23 & $33.57 \pm 1.22$ & 68 \\
\hline cyclo(vLwIL) 24 & $21.22 \pm 0.93$ & 43 \\
\hline cyclo(vLflL) 1 & $92.04 \pm 17.86$ & 186 \\
cyclo(vLwIL) 24 & $9.48 \pm 2.51$ & 19 \\
\hline cyclo(vLsIL) 25 & $2.90 \pm 0.32$ & 6
\end{tabular}

b

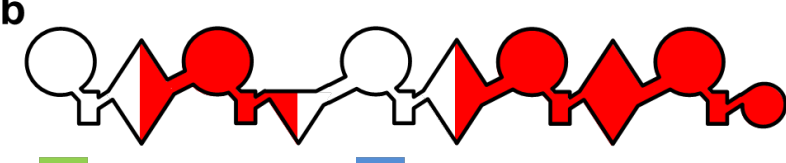

\begin{tabular}{rcc}
\multicolumn{1}{c}{ Peptide } & $\begin{array}{c}\text { Production } \\
\text { [mg/l] }\end{array}$ & $\begin{array}{c}\text { \% of WT } \\
\text { Level (1) }\end{array}$ \\
\hline iLmL 26 & $0.79 \pm 0.01$ & 1.61 \\
iLwL 27 & $4.35 \pm 0.1$ & 8.88 \\
vLwL 28 & $1.36 \pm 0.01$ & 2.78 \\
vLIL 13 & $0.73 \pm 0.01$ & 1.49 \\
iLfL 29 & $9.85 \pm 0.2$ & 20.1 \\
vLfL 11 & $2.22 \pm 0.13$ & 4.53 \\
iLIL 30 & $1.02 \pm 0.06$ & 2.08 \\
\hline LwIL 31 & $1.05 \pm 0.03$ & 2.14 \\
cyclo[rLwIL] 32 & $17.65 \pm 1.28$ & 36.1 \\
\hline cyclo[sLtIL] 33 & $0.16 \pm 0.04$ & 0.33 \\
\hline cyclo(vLflL) 1 & $122.37 \pm 13.95$ & 249 \\
vLflL 5 & $0.23 \pm 0.02$ & 0.47 \\
cyclo[vLwIL] 24 & $9.31 \pm 1.62$ & 19.0 \\
vLwIL 34 & $0.11 \pm 0.01$ & 0.22 \\
vLIIL 35 & $0.09 \pm 0.02$ & 0.18 \\
LfIL 36 & $0.98 \pm 0.04$ & 2.0 \\
LwIL 31 & $0.87 \pm 0.01$ & 1.78 \\
vLIL 13 & $0.69 \pm 0.01$ & 1.41
\end{tabular}

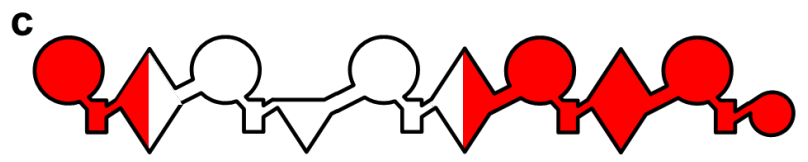

\begin{tabular}{rcc}
\multicolumn{1}{c}{ Peptide } & $\begin{array}{c}\text { Production } \\
{[\mathrm{mg} / / \mathrm{l}]}\end{array}$ & $\begin{array}{c}\text { \% of WT } \\
\text { Level (1) }\end{array}$ \\
\hline cyclo(vLflL) 1 & $0.19 \pm 0.06$ & 0.4 \\
\hline LwlL 31 & $3.34 \pm 0.07$ & 6.8 \\
cyclo(vLwlL) 24 & $16.84 \pm 1.29$ & 34 \\
VLwlL 37 & $1.47 \pm 0.19$ & 3 \\
LLwIL 38 & $0.38 \pm 0.04$ & 0.8 \\
vLwlL 34 & $0.15 \pm 0.01$ & 0.3 \\
cyclo(ILwIL) 39 & $3.15 \pm 0.10$ & 6.4 \\
\hline cyclo(vLrlL) 23 & $4.18 \pm 0.27$ & 8.5
\end{tabular}

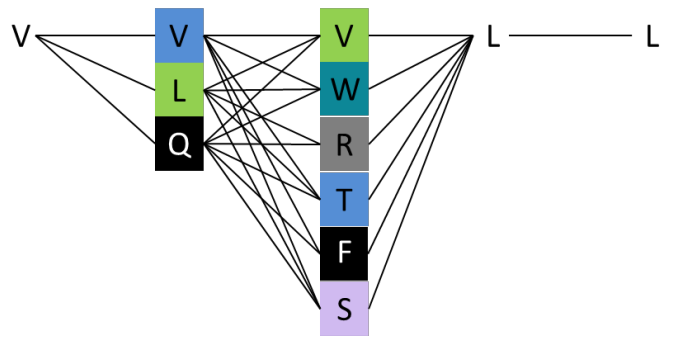

Kols BicA Xeys Xtps Xenolindicin-like
Pax AmbS $_{\text {indica }} \bigcirc$ AmbS $_{\text {miraniensis }} \bigcirc$ Gxps

Figure 4. Targeted randomization of GxpS. Schematic representation of all possible recombinant NRPSs and corresponding NRPs (left). Detected peptides and corresponding peptide yields (right) as obtained from triplicate experiments. For peptide nomenclature the standard one letter AA code with lowercase for D-AA is used. For assignment of domain symbols see Fig. 1. Bottom: Color code of NRPS used as building blocks (for details see Supplementary Figure 5). (a) Randomization of position three from GxpS. (b) Randomization of position one and three from GxpS. (c) Randomization of adjacent positions two and three. 